\title{
Peak exercise capacity estimated from incremental shuttle walking test in patients with COPD: a methodological study Ragnheiður Harpa Arnardóttir*1, Margareta Emtner ${ }^{1}$, Hans Hedenström², Kjell Larsson ${ }^{3}$ and Gunnar Boman ${ }^{1}$
}

Address: ${ }^{1}$ Department of Medical Sciences: Respiratory Medicine and Allergology, Uppsala University, Uppsala, Sweden, ${ }^{2}$ Department of Medical Sciences: Clinical Physiology, Uppsala University, Uppsala, Sweden and ${ }^{3}$ Lung and Allergy Research, National Institute of Environmental Medicine, Karolinska Institutet, Stockholm, Sweden

Email: Ragnheiður Harpa Arnardóttir* - harpa.arnardottir@medsci.uu.se; Margareta Emtner - margareta.emtner@sjukgym.uu.se; Hans Hedenström - hans.hedenstrom@akademiska.se; Kjell Larsson - kjell.larsson@imm.ki.se; Gunnar Boman - gunnar.boman@medsci.uu.se

* Corresponding author

Published: 17 October 2006

Respiratory Research 2006, 7:127 doi:10.1 186/1465-9921-7-127
Received: 31 May 2006

Accepted: 17 October 2006

This article is available from: http://respiratory-research.com/content/7/I/I27

(C) 2006 Arnardóttir et al; licensee BioMed Central Ltd.

This is an Open Access article distributed under the terms of the Creative Commons Attribution License (http://creativecommons.org/licenses/by/2.0), which permits unrestricted use, distribution, and reproduction in any medium, provided the original work is properly cited.

\begin{abstract}
Background: In patients with COPD, both laboratory exercise tests and field walking tests are used to assess physical performance. In laboratory tests, peak exercise capacity in watts (W peak) and/or peak oxygen uptake $\left(\mathrm{VO}_{2}\right.$ peak) are assessed, whereas the performance on walking tests usually is expressed as distance walked. The aim of the study was to investigate the relationship between an incremental shuttle walking test (ISWT) and two laboratory cycle tests in order to assess whether $\mathrm{W}$ peak could be estimated from an ISWT.
\end{abstract}

Methods: Ninety-three patients with moderate or severe COPD performed an ISWT, an incremental cycle test (ICT) to measure W peak and a semi-steady-state cycle test with breath-bybreath gas exchange analysis (CPET) to measure $\mathrm{VO}_{2}$ peak. Routine equations for conversion between cycle tests were used to estimate $\mathrm{W}$ peak from measured $\mathrm{VO}_{2}$ peak (CPET). Conversion equation for estimation of W peak from ISWT was found by univariate regression.

Results: There was a significant correlation between $W$ peak and distance walked on ISWT $\times$ body weight $(r=0.88, p<0.000 I)$. The agreement between $W$ peak measured by ICT and estimated from ISWT was similar to the agreement between measured W peak (ICT) and W peak estimated from measured $\mathrm{VO}_{2}$ peak by CPET.

Conclusion: Peak exercise capacity measured by an incremental cycle test could be estimated from an ISWT with similar accuracy as when estimated from peak oxygen uptake in patients with COPD.

\section{Background}

Measurements of exercise capacity are important and widely used in rehabilitation of patients with chronic obstructive pulmonary disease (COPD). Exercise testing in COPD varies from maximal laboratory tests, requiring advanced technical equipment, to simple field tests. Maximal laboratory tests are mostly constructed to measure peak exercise capacity (W peak), and/or peak oxygen uptake $\left(\mathrm{VO}_{2}\right.$ peak) whereas field tests have been considered to reflect functional capacity [1-3]. The incremental 
shuttle walking test (ISWT) is a field test which is similar to the laboratory tests as it is externally paced and progressive [4]. During ISWT there is a linear relationship between $\mathrm{VO}_{2}$ and walking speed, similar to the relationship between $\mathrm{VO}_{2}$ and work rate in incremental laboratory testing $[5,6]$. Singh et al found, when comparing incremental laboratory treadmill test and ISWT, that $\mathrm{VO}_{2}$ peak could be estimated from distance walked on ISWT [5].

Singh et al compared two different walking tests (treadmill and ISWT) and it is unclear whether such a strong relationship would also be found between ISWT and cycle performance. In laboratory testing, the treadmill test evokes slightly higher ratings of $\mathrm{VO}_{2}$ peak than the cycle tests $[7,8]$, whereas different protocols of cycle tests usually result in similar $\mathrm{VO}_{2}$ peak but different $\mathrm{W}$ peak, depending on the slope of increased load during the test [9-11]. Body weight is an important contributor to the work load during walking, whereas it is of minor importance during cycling. Thus, the correlation between performance on timed walking tests and $\mathrm{VO}_{2}$ peak from a cycle test becomes stronger if distance walked is multiplied by body weight (distance $\times$ weight $=$ work of walking at horizontal level) $[12,13]$. Recent findings indicate that metabolic and ventilatory responses to walking may differ from the responses to cycling in patients with COPD $[8,14,15]$. In pulmonary rehabilitation many exercise programmes are conducted on ergometer cycles. Target training intensity is often expressed as a percent of $\mathrm{W}$ peak, which in turn usually is defined as the highest work rate tolerated during an incremental cycle test with 1-minute steps or ramp increments. From a known $\mathrm{VO}_{2}$ peak it is possible to estimate $\mathrm{W}$ peak $[16,17]$, and as $\mathrm{VO}_{2}$ peak can be estimated from an ISWT [5] it seems reasonable to assume that $\mathrm{W}$ peak could be estimated from ISWT through the estimated $\mathrm{VO}_{2}$ peak. However, using two conversion equations would make the results less reliable. Therefore, an estimation of $\mathrm{W}$ peak directly from the performance on ISWT would be preferable. This would be of clinical interest when expensive laboratory tests are not available.

The aim of the present study was to investigate whether $\mathrm{W}$ peak (assessed on a cycle ergometer) could be estimated from an ISWT in patients with moderate or severe COPD. For this purpose, comparisons of ISWT and two different cycle tests were made.

\section{Methods \\ Material}

Ninety-three subjects with moderate or severe COPD according to the British Thoracic Society [18] were consecutively invited to take part in the study when being referred for training to the Physiotherapy Unit of the Pul- monary Section at the Akademiska Hospital, Uppsala, Sweden during 2001-2004. All were smokers or ex-smokers. The study was approved by the Medical Ethics Committee of Uppsala University and all subjects gave informed consent.

Inclusion criteria were COPD with forced expiratory volume in one second $\left(\mathrm{FEV}_{1}\right)<60 \%$ of predicted value and $\mathrm{FEV}_{1} / \mathrm{VC}$ (vital capacity) $<0.7$ after bronchodilatation [18]. Exclusion criteria were other diseases interfering with exercise such as ischemic coronary disease and musculo-skeletal problems.

\section{Testing}

Lung function was measured with a Masterlab Trans spirometer, Masterlab Body Plethysmograph and Masterlab Transfer (Erich Jaeger AG, Würzburg, Germany) in accordance with the ATS guidelines [19]. Swedish reference values were used $[20,21]$.

Incremental shuttle walking test (ISWT) was performed in a level corridor. Two cones were placed $9 \mathrm{~m}$ apart comprising a $10 \mathrm{~m}$ track as described by Singh et al [4]. Instructions to the subject and the pace of the test were played from a tape recorder. The test commenced at a speed of $30 \mathrm{~m} / \mathrm{min}$ which then was increased by $10 \mathrm{~m} /$ min every minute. The subjects continued walking until they were not able to reach the next cone in time for the signal or got too exhausted to continue. The total distance walked was the main outcome of the test. Before and directly after walking, oxygen saturation $\left(\mathrm{SpO}_{2}\right)$, heart rate, peak expiratory flow (PEF), perceived exertion (Borg RPE-scale) [22] and dyspnoea (Borg CR-10-scale) [23] were registered. The patients carried a pulse oximeter (Optovent Respons, Optovent, Linköping, Sweden) by a shoulder strap throughout the test. In 52 of the patients the test was identically repeated within a week.

Symptom-limited incremental cycle ergometer test (ICT) (Case 8000 Exercise Testing System, GE Medical Systems, Milwaukee, USA) with continuous ECG-registration was conducted to measure peak work load (W peak). The subjects started pedalling at $20 \mathrm{~W}$ and the load was then increased by $10 \mathrm{~W}$ every fulfilled minute until exhaustion. Heart rate, breathing frequency and $\mathrm{SpO}_{2}$ (Optovent Respons) were registered every minute during exercise. Systolic blood pressure, subjective ratings of perceived exertion and dyspnoea were recorded every other minute $[22,23]$. All variables were measured before and 1, 2, 4 and 10 minutes after exercise.

A semi-steady-state cardiopulmonary exercise test with breath-by-breath gas exchange analysis (CPET) was performed, according to routines at our clinic, after resting for at least $30 \mathrm{~min}$ after the incremental cycle test (longer 
Table I: Characteristics of the patients. Values expressed as mean $\pm S D$ and (range), $n=93$.

\begin{tabular}{|c|c|c|c|}
\hline Gender, male/female & $26 / 67$ & & \\
\hline Smoking habits, current/ex & $23 / 70$ & & \\
\hline Age, years & 64 & \pm 7 & $(43-80)$ \\
\hline Weight, kg & 65.4 & \pm 13.2 & $(42-101)$ \\
\hline BMI, kg/m² & 23.4 & \pm 4.3 & $(14.5-34.9)$ \\
\hline Packyears & 36 & \pm 21 & $(8-120)$ \\
\hline VC, liters & 2.6 & \pm 0.9 & $(1.3-6.1)$ \\
\hline VC, \% predicted & 67 & \pm 16 & $(40-121)$ \\
\hline $\mathrm{FEV}_{1}$, liters & 0.9 & \pm 0.3 & $(0.4-1.7)$ \\
\hline $\mathrm{FEV}_{1}, \%$ predicted & 32 & \pm 11 & $(14-59)$ \\
\hline MVV, liters/min & 33.5 & \pm 12.7 & $(15.0-66.7)$ \\
\hline MVV, \% predicted & 46 & \pm 12 & $(16-83)$ \\
\hline $\mathrm{DLCO}, \mu \mathrm{mol} / \mathrm{sec} / \mathrm{kPa}$ & 57.2 & \pm 22.4 & $(16.2-127)$ \\
\hline DLCO, \% predicted & 50 & \pm 18 & $(15-106)$ \\
\hline
\end{tabular}

BMI: body mass index; VC: vital capacity; $\mathrm{FEV}_{1}$ : forced expiratory volume in one second; MVV: maximum voluntary ventilation; DLCO: carbon monoxide diffusion capacity.

if needed for all resting parameters to be stable at pre-exercise levels). Measurements of heart rate, $\mathrm{SpO}_{2}$ and ratings of perceived exertion and dyspnoea were made identically to the incremental cycle test. To enable measurement of $\mathrm{VO}_{2}$ peak, the subjects wore a mask with a turbine for gas exchange analysis (Oxycon Sigma, Jaeger, Germany). Additionally, $\mathrm{VCO}_{2}$ (carbon dioxide), minute ventilation $\left(\mathrm{V}_{\mathrm{E}}\right)$, respiratory quotient $(\mathrm{RQ})$ and breathing frequency were measured with readings every 30 seconds. After recording steady-state measurements at rest (approximately 4 min of registration at rest) the patient started pedalling at $20 \mathrm{~W}$. The load was kept constant until the ventilation and oxygen uptake reached a plateau, on average 3-4 min at each level, then the load was increased. To keep testing time within reasonable limits the load was increased until exhaustion by $5,10,20$ or $30 \mathrm{~W}$ depending on the outcome of the first test (ICT).

The reason for discontinuing the cycle tests and the ISWT was stated at the end of each test.

The two different ergometer cycle tests were performed on the same day, but the lung function test and the ISWT were conducted on separate days. The three test days were separated by $1-3$ resting days.

\section{Conversion equations}

$\mathrm{W}$ peak was estimated from the measured $\mathrm{VO}_{2}$ peak on CPET by an equation derived from Åstrand [16]: $\left(\mathrm{VO}_{2}\right.$ peak $\left.\times 1000^{-1}-0.1517\right) \times 0.0134^{-1}$. The equation from Singh et al for estimating $\mathrm{VO}_{2}$ from ISWT [5] was multiplied by body weight to express $\mathrm{ml} / \mathrm{min}$ instead of $\mathrm{ml} /$ $\mathrm{min} / \mathrm{kg}$ : $(4.19+0.025 \times$ walking distance $) \times$ body weight.

\section{Statistical analysis}

Results were expressed as mean and standard deviation (SD) or 95\% confidence interval (CI). For simple correlations Pearson's correlation coefficient was calculated. ANOVA and Student's $t$-test were calculated for paired comparisons, except for RPE and CR-10 scores where Friedman's ANOVA and Wilcoxon's signed rank test were used. Scatterplots as recommended by Bland and Altman were made for comparison of estimated and measured values. The level of significance was set at $5 \%$.

\section{Results}

All 93 subjects accepted to participate and were enrolled in the study, 71 with severe disease according to the British Thoracic Society guidelines [18]. There were no dropouts. See Table 1 for characteristics.

\section{Test results}

Distance walked on ISWT was 314 (291-336) m. Endexercise work load was $62(57-68) \mathrm{W}$ on the incremental cycle test (i.e. W peak) and $46(41-50) \mathrm{W}$ on the CPET. Measured peak $\mathrm{VO}_{2}$ on CPET was $973(908-1038) \mathrm{ml} /$ $\mathrm{min}$. There was significantly lower peak heart rate, $\mathrm{SpO}_{2}$, ratings of perceived exertion and dyspnoea at the end of the walking test compared to the cycle tests (Table 2). The reported reasons for cessation were identical in the two cycle tests: $39 \%$ because of dyspnoea, 35\% because of a combination of dyspnoea and exertion, $20 \%$ because of exertion and $6 \%$ because of leg fatigue. In the ISWT the reason for cessation was dyspnoea in 3\%, a combination of dyspnoea and exertion in $12 \%$ and inability to increase or keep up the speed to reach the next cone in time in $85 \%$ of the cases.

Fifty-two subjects repeated the ISWT within a week. The difference between the two tests was not significant for

Table 2: End-exercise values from the three tests: ISWT, ICT and CPET, $\mathbf{n}=93$.

\begin{tabular}{|c|c|c|c|c|c|c|}
\hline & ISWT & & $\mathrm{ICT}$ & & CPET & \\
\hline $\mathrm{SpO}_{2,} \%$ & $87 * * *$ & $(86-88)$ & 90 & $(89-91)$ & $89+$ & $(88-91)$ \\
\hline Heart rate, beats/min & $115^{* * * *}$ & $(111-118)$ & $13 \mid$ & $(127-138)$ & $13 \mid$ & $(124-134)$ \\
\hline Perceived exertion, RPE & 15.9 **** & $(15.5-16.3)$ & 17.4 & $(17.1-17.6)$ & 17.5 & $(17.2-17.7)$ \\
\hline Dyspnoea, CR-10 & $6.2 * * *$ & $(5.8-6.6)$ & 7.9 & $(7.6-8.3)$ & 8.0 & $(7.7-8.4)$ \\
\hline
\end{tabular}

ISWT: incremental shuttle walking test, ICT: incremental cycle test, CPET: semi steady-state cardiopulmonary exercise test, $\mathrm{SPO}_{2}$ : oxygen saturation in blood measured by pulsoximeter, RPE: Borg scale for ratings of perceived exertion, CR-10: Borg scale for dyspnoea, VO $\mathrm{O}_{2}$ peak: peak oxygen uptake, ***: $\mathrm{p}<0.000 \mathrm{I}$ compared to ICT and CPET, t: $\mathrm{p}<0.05$ compared to ICT. Mean $(95 \% \mathrm{Cl})$. 
W peak (watt)

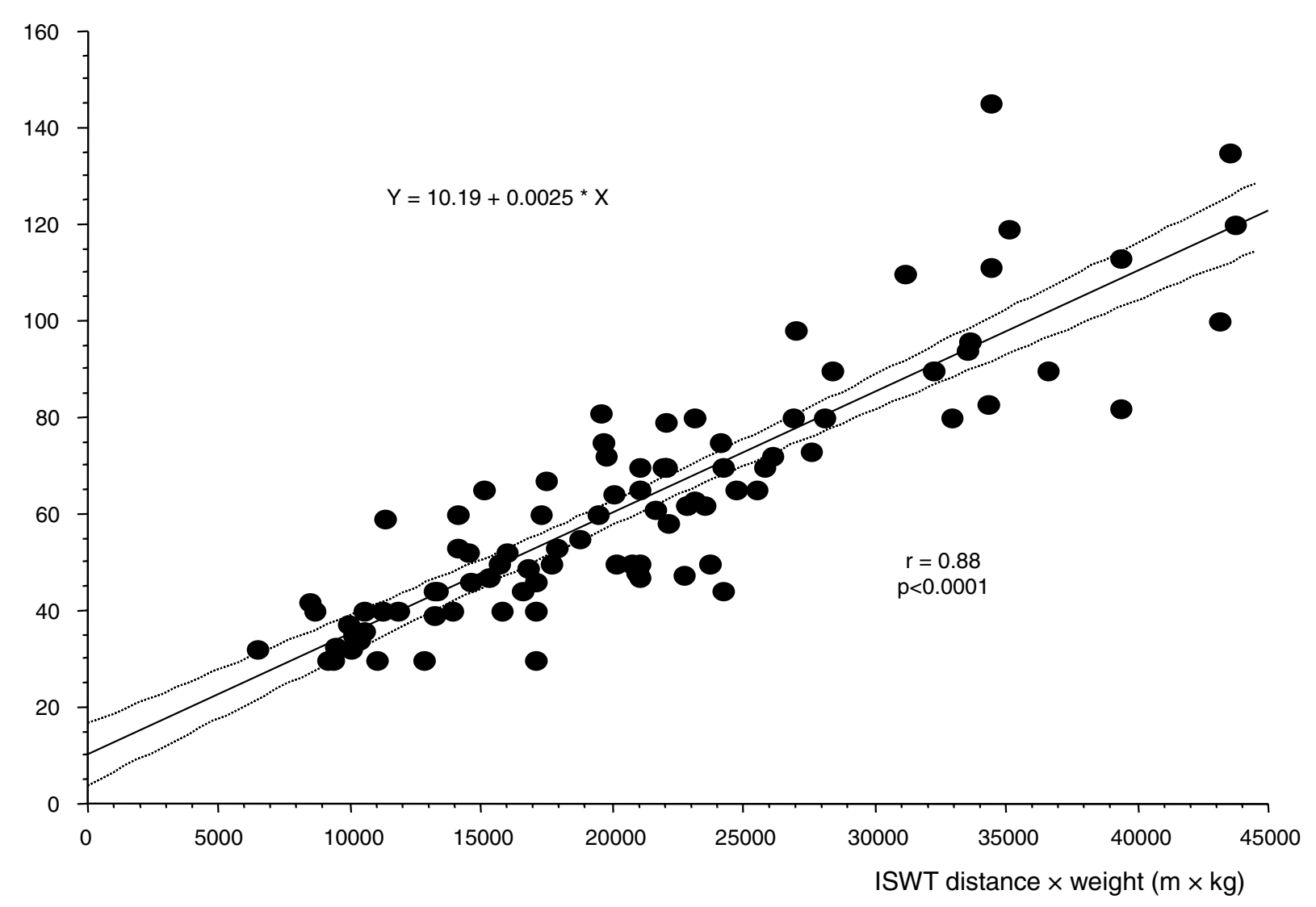

Figure I

The correlation between the peak exercise capacity (W peak) measured by an incremental cycle ergometer test and the distance walked $(\mathrm{m})$ on an incremental shuttle walking test (ISWT) multiplied by body weight $(\mathrm{kg})$. Regression line and $95 \%$ confidence bands. $n=93$

any variable measured, mean difference in walking distance being $9 \pm 38 \mathrm{~m}$ or $3 \% \pm 12 \%(\mathrm{p}=0.09)$. All calculations were therefore based on the first ISWT. This subgroup did not differ from the larger group in any baseline characteristics.

\section{Correlations and estimated $W$ peak}

There was a significant correlation $(\mathrm{r}=0.88$, $\mathrm{p}<0.0001)$ between ISWT distance $\times$ body weight and the measured $\mathrm{W}$ peak from the incremental cycle test: $\mathrm{W}$ peak $=0.0025$ $\times$ distance $(\mathrm{m}) \times$ body weight $(\mathrm{kg})+10.19$ (Fig. 1). W peak estimated from performance on ISWT by this equation was 62 (57-66) W and did not differ significantly from the measured $\mathrm{W}$ peak $(62(57-68) \mathrm{W}, \mathrm{p}=0.7)$. For comparison, $\mathrm{W}$ peak was estimated from the measured $\mathrm{VO}_{2}$ peak by the equation derived from Åstrand [16], resulting in an estimated $\mathrm{W}$ peak of 61 (56-66) W. This was not significantly different from the measured $\mathrm{W}$ peak or the estimated W peak from ISWT above. A strong agreement was found between the measured and estimated values of $\mathrm{W}$ peak with a minor tendency to overestimation at the lower range and underestimation at the higher range of performance both when estimated from ISWT and from $\mathrm{VO}_{2}$ peak (Fig. 2). There is one outlier where ISWT clearly underestimated W peak (Fig. 2). This was the only subject who would have been able to run at the end of the ISWT, which, however, is not allowed in a walk test.

No significant differences were found between women and men regarding the relationship between the different exercise tests.

\section{Comparisons with estimation based on previous findings}

To test whether $\mathrm{W}$ peak could be estimated by use of the equation by Singh et al [5], we estimated $\mathrm{VO}_{2}$ peak from their equation and then converted this estimated $\mathrm{VO}_{2}$ to 

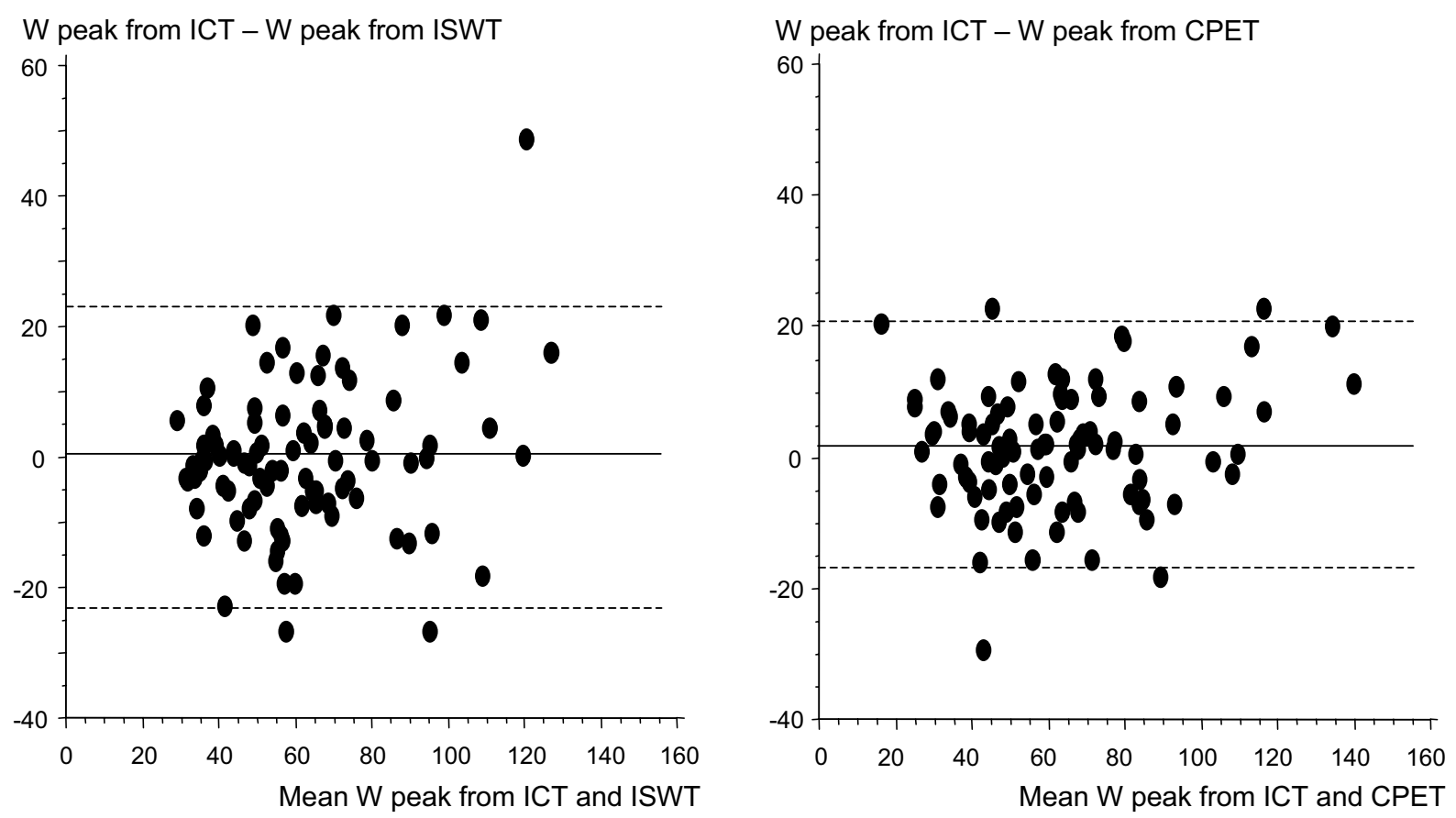

Figure 2

Scatterplots (Bland-Altman). On the left, the difference between measured maximum exercise capacity (W peak) measured by incremental cycle test (ICT) and estimated $W$ peak from performance on incremental shuttle walking test (ISWT) against the mean values of those two. On the right, the difference between measured $W$ peak from ICT and estimated $W$ peak from measured maximum oxygen uptake on semi steady-state cardiopulmonary exercise test (CPET) [16] against the mean of those two. Whole line: mean difference, dotted lines: $\pm 2 S D$.

estimated $\mathrm{W}$ peak (as above) [16]. Using the equation by Singh et al, $\mathrm{VO}_{2}$ peak was underestimated compared to our measurements $(-171 \pm 58 \mathrm{ml} / \mathrm{min}$ or $-16 \pm 15 \%$, p < $0.0001)$ which consequently resulted in an underestimation of $\mathrm{W}$ peak $(-14 \pm 13 \mathrm{~W}, \mathrm{p}<0.0001)$.

\section{Discussion}

In the present study, ISWT distance $\times$ body weight was a good predictor of $\mathrm{W}$ peak measured by an incremental cycle test in patients with moderate or severe COPD. The fact that W peak estimated from ISWT was as accurate as $\mathrm{W}$ peak estimated from $\mathrm{VO}_{2}$ is of clinical importance, as the ISWT is much simpler and cheaper than a laboratory cycle test.

Our results confirm the findings of others that there is an excellent correlation between performance on ISWT and laboratory testing $[5,6,24]$. Although the correlation in our study between $\mathrm{VO}_{2}$ and distance walked was almost identical with the findings of Singh et al [5], applying their equation in our material resulted in a significant underestimation. One likely explanation to this could be the difference in the number of subjects, as in their study 19 subjects were included, compared to 93 subjects in the current study. As Singh et al performed two ISWT, and used the second test in their analysis, that might explain some of the inconsistency between their equation and ours, even though no significant improvement on retesting was found in our material. The majority of patients in the study by Singh et al were men while our study was dominated by women. Gender did not significantly affect the relationship between the different tests in our analysis, but this might need further investigation, as only $26 \%$ of our subjects were men. The fact that Singh et al compared two walking tests whereas we compared walking and cycling might also explain the difference to some extent, as there is a known difference in metabolic adaptations during walking and cycling $[8,15]$. However, as $\mathrm{VO}_{2}$ peak measured on a treadmill test has been found to be higher than [8] or equal to [25] $\mathrm{VO}_{2}$ peak measured on a cycle test in patients with COPD, a regression equation derived from a treadmill test could be expected to overestimate $\mathrm{VO}_{2}$ peak on a cycle rather than the opposite.

All patients who were referred to pulmonary rehabilitation and fulfilled the inclusion criteria during the time of the study agreed to participate. Therefore, we conclude that the study is representative of a COPD-population 
referred to rehabilitation including physical training. The wide range of lung function impairment and age of the participants also enhances the relevance of our sample. In Sweden, women have caught up with men in the prevalence of and mortality in COPD [26]. The majority of women referred to pulmonary rehabilitation during the time of the study is noticeable and we can only speculate that this might indicate that women were either more often offered referral to pulmonary rehabilitation by their physicians or were more likely to accept such an offer than the men.

Heart rate, RPE- and CR-10-scores were significantly lower in ISWT than in the cycle tests. The cycle tests were mainly limited by breathlessness and/or exertion whereas the ISWT was, in most cases, limited by the incapability to increase the speed of walking. During walking it is difficult to increase walking speed above a certain level. Some treadmill test protocols are therefore constructed to increase inclination rather than speed [27].

$\mathrm{SpO}_{2}$ decreased more by walking than cycling in the current study, quite in line with previous findings $[8,14,24]$. It has been speculated that the positional differences between walking and cycling could lead to less effective breathing during walking and thus more desaturation [15]. In spite of the above differences between walking and cycling, our results demonstrated that in patients with moderate to severe COPD it was possible to estimate cycle performance from an ISWT quite as accurately as when estimating performance from one laboratory cycle test to the other. As equations work both ways, our findings also make it possible to estimate distance walked (and thereby walking speed) on ISWT from W peak. This could be useful in clinics where laboratory cycle testing is routine practice but exercise training prescription is mainly walking. As the outlier in our study illustrates, the ISWT can be expected to mimic performance in a symptom-limited cycle test only in subjects that reach their exercise limit by brisk walk, i.e. are unable to run, as otherwise the subjects would not be close to their peak capacity during the test. Being able to run is, however, a very rare condition in COPD-patients referred to rehabilitation, implying that this does not undermine the use of ISWT in patients with COPD.

We used the first ISWT (no training test) for our analyses. Due to the patient's poor condition or because of time constraints it is often not feasible to perform a training test in clinical practice. Thus, it was clinically relevant to present our calculations based on the first ISWT. This was also supported by the finding that the walking distance did not increase at the second test performed within a week. Control calculations were done by using results (not shown) from the second ISWT $(\mathrm{n}=52)$ in our mate- rial and no differences were found. Significant difference in walking distance has previously been found on repeated testing with ISWT in patients with COPD $[4,28]$. It is not clear why our results are inconsistent with previous studies regarding the repeatability of ISWT, but, as noted above, the number of subjects and gender distribution is somewhat different from the other studies, and this might affect the repeatability.

It could be argued that the cycle tests should have been performed in random order. The present design was chosen because it enabled us to adjust the progressive pattern of the CPET to keep exercise time within reasonable limits when $\mathrm{W}$ peak from the incremental cycle test was known. Although all subjects rested between the tests, we can not rule out the possibility that the order of the tests could have influenced the results. However, as peak heart rate, CR-10 scores and ratings of perceived exertion (RPE) were almost identical in the two cycle tests, and W peak could be estimated from $\mathrm{VO} 2$ peak as expected [16], we consider this error as relatively small.

Being able to estimate W peak by ISWT for clinical purposes does not make ISWT a perfect substitute for the incremental cycle test or other forms of laboratory tests. There is, of course, some variation between estimated and measured peak values on an individual basis, but most importantly the safety aspect of the laboratory tests is beyond the ISWT. Therefore, for COPD patients considered at increased risk for cardiovascular hazards, patients that have never had proper cardiologic assessment or patients with uncertain diagnosis, laboratory tests must be considered as the first choice. However, for COPDpatients of minor risk of cardiovascular incidents, and where laboratory tests are scarce, the ISWT could be used as an alternative for estimating $\mathrm{W}$ peak.

\section{Conclusion}

Peak exercise capacity measured by an incremental cycle test could be estimated from an ISWT with similar accuracy as when estimated from peak oxygen uptake.

\section{Competing interests}

The author(s) declare that they have no competing interests.

\section{Authors' contributions}

All authors participated in the design of the study and writing the manuscript as well as approving the final manuscript. HH and RHA participated in data collection, RHA drafted the manuscript.

\section{Acknowledgements}

Special thanks to Ulrike Spetz-Nyström for her valuable help with testing. This paper was supported by grants from the Swedish Heart-Lung Founda- 
tion, The County Council of Uppsala and various funds at Uppsala University.

\section{References}

I. Clinical exercise testing with reference to lung diseases: indications, standardization and interpretation strategies. ERS Task Force on Standardization of Clinical Exercise Testing. European Respiratory Society. Eur Respir J 1997, I O(I I):2662-2689.

2. ATS statement: guidelines for the six-minute walk test. $A m \mathrm{~J}$ Respir Crit Care Med 2002, I 66(I): I I I-II7.

3. McGavin CR, Gupta SP, McHardy GJ: Twelve-minute walking test for assessing disability in chronic bronchitis. $\mathrm{Br}$ Med J I976, I (60 I 3):822-823.

4. Singh SJ, Morgan MD, Scott S, Walters D, Hardman AE: Development of a shuttle walking test of disability in patients with chronic airways obstruction. Thorax 1992, 47(12): 1019-1024.

5. Singh SJ, Morgan MD, Hardman AE, Rowe C, Bardsley PA: Comparison of oxygen uptake during a conventional treadmill test and the shuttle walking test in chronic airflow limitation. Eur Respir J 1994, 7( I I):2016-2020.

6. Onorati P, Antonucci R, Valli G, Berton E, De Marco F, Serra P, Palange $P$ : Non-invasive evaluation of gas exchange during a shuttle walking test vs. a 6-min walking test to assess exercise tolerance in COPD patients. Eur J Appl Physiol 2003, 89(34):331-336.

7. Page E, Cohen-Solal A, Jondeau G, Douard H, Roul G, Kantelip JP, Bussiere JL: Comparison of treadmill and bicycle exercise in patients with chronic heart failure. Chest 1994, I06(4): I002-1006.

8. Christensen CC, Ryg MS, Edvardsen A, Skjonsberg OH: Effect of exercise mode on oxygen uptake and blood gases in COPD patients. Respir Med 2004, 98(7):656-660.

9. Myers J, Buchanan N, Walsh D, Kraemer M, McAuley P, HamiltonWessler M, Froelicher VF: Comparison of the ramp versus standard exercise protocols. J Am Coll Cardiol 199I, I 7(6): | 334- I 342.

10. Zhang YY, Johnson MC 2nd, Chow N, Wasserman K: Effect of exercise testing protocol on parameters of aerobic function. Med Sci Sports Exerc 1991, 23(5):625-630.

II. Revill SM, Beck KE, Morgan MD: Comparison of the peak exercise response measured by the ramp and I-min step cycle exercise protocols in patients with exertional dyspnea. Chest 2002, I 2 I(4): I099-I I05.

12. Chuang ML, Lin IF, Wasserman K: The body weight-walking distance product as related to lung function, anaerobic threshold and peak VO2 in COPD patients. Respir Med 200I, 95(7):618-626.

13. Carter R, Holiday DB, Stocks J, Grothues C, Tiep B: Predicting oxygen uptake for men and women with moderate to severe chronic obstructive pulmonary disease. Arch Phys Med Rehabil 2003, 84(8): I I58-I I64.

14. Man WD, Soliman MG, Gearing J, Radford SG, Rafferty GF, Gray BJ, Polkey MI, Moxham J: Symptoms and quadriceps fatigability after walking and cycling in chronic obstructive pulmonary disease. Am J Respir Crit Care Med 2003, I 68(5):562-567.

15. Palange P, Forte S, Onorati P, Manfredi F, Serra P, Carlone S: Ventilatory and metabolic adaptations to walking and cycling in patients with COPD. J Appl Physiol 2000, 88(5): I $715-1720$.

16. Åstrand PO, Rodahl K: Textbook of work physiology: Physiological Bases of Exercise. 3rd edition. New York, McGraw-Hill; 1986.

17. Wallin L, Brudin LH: Physical working capacity determined by different types of bicycle exercise tests. Clin Physiol 1988, 8(5):529-537.

18. BTS guidelines for the management of chronic obstructive pulmonary disease. The COPD Guidelines Group of the Standards of Care Committee of the BTS. Thorax 1997, 52 Suppl 5:SI-28.

19. Standardization of spirometry-- 1987 update. Official statement of American Thoracic Society. Respir Care 1987, 32(I I): 1039-1060.

20. Hedenstrom H, Malmberg P, Agarwal K: Reference values for lung function tests in females. Regression equations with smoking variables. Bull Eur Physiopathol Respir 1985, 2 I (6):55 I-557.
21. Hedenstrom H, Malmberg P, Fridriksson HV: Reference values for lung function tests in men: regression equations with smoking variables. Ups J Med Sci 1986, 9 I(3):299-310.

22. Borg G: Perceived exertion as an indicator of somatic stress. Scand J Rehabil Med 1970, 2(2):92-98.

23. Borg GA: Psychophysical bases of perceived exertion. Med Sci Sports Exerc 1982, I 4(5):377-38I.

24. Turner SE, Eastwood PR, Cecins NM, Hillman DR, Jenkins SC: Physiologic responses to incremental and self-paced exercise in COPD: a comparison of three tests. Chest 2004, I 26(3):766-773.

25. Mathur RS, Revill SM, Vara DD, Walton R, Morgan MD: Comparison of peak oxygen consumption during cycle and treadmill exercise in severe chronic obstructive pulmonary disease. Thorax 1995, 50(8):829-833.

26. [http://www.sos.se/epc/epceng.htm\#epid]

27. Nagle FJ, Balke B, Naughton JP: Gradational step tests for assessing work capacity. J Appl Physiol 1965, 20(4):745-748.

28. Eiser N, Willsher D, Dore CJ: Reliability, repeatability and sensitivity to change of externally and self-paced walking tests in COPD patients. Respir Med 2003, 97(4):407-4I4.
Publish with Bio Med Central and every scientist can read your work free of charge

"BioMed Central will be the most significant development for disseminating the results of biomedical research in our lifetime. "

Sir Paul Nurse, Cancer Research UK

Your research papers will be:

- available free of charge to the entire biomedical community

- peer reviewed and published immediately upon acceptance

- cited in PubMed and archived on PubMed Central

- yours - you keep the copyright

Submit your manuscript here:

http://www.biomedcentral.com/info/publishing_adv.asp
BioMedcentral 